\title{
Acute and Chronic Effects of Combined Training on Brain-Derived Neurotrophic Factor Levels and Its Association with Anthropometric Variables in Overweight Men
}

\author{
Yazgaldi Nazari, ${ }^{1, *}$ Masoud Nikbakht, ${ }^{2}$ Abdolhamid Habibi, ${ }^{3}$ and Saeed Shakeryan ${ }^{4}$ \\ ${ }^{1} \mathrm{Ph} . \mathrm{D}$ student in Exercise Physiology, Faculty of Physical Education and Sport Sciences, Shahid Chamran University of Ahvaz, Ahvaz, Iran \\ ${ }^{2}$ Associate Professor of Exercise Physiology, Shahid Chamran University of Ahvaz, Ahvaz, Iran \\ ${ }^{3}$ Professor of Exercise Physiology, Shahid Chamran University of Ahvaz, Ahvaz, Iran \\ ${ }^{4}$ Assistant Professor of Exercise Physiology, Shahid Chamran University of Ahvaz, Ahvaz, Iran \\ "Corresponding author: Yazgaldi Nazari, Faculty of Physical Education and Sport Sciences, Shahid Chamran University of Ahvaz, Ahvaz, Iran. Tel: +98-9364154977, E-mail: \\ y.nazari53@gmail.com
}

Received 2016 October 16; Revised 2016 November 07; Accepted 2016 December 12.

\begin{abstract}
Objectives: Physical activity, one of the most important factors affecting energy metabolism, plays an important role against obesity and being overwight. Brain-derived neurotrophic factor, in addition to its role in the differentiation of neurons and synaptic plasticity, has a key role in controlling body weight and energy homeostasis through hypothalamus pathway. Therefore, this study addressed acute and chronic effects of combined training on brain-derived neurotrophic factor levels. It also probed the relationship between brain-derived neurotrophic factors and anthropometric variables including body mass index and fat percentage in overweight young men.

Methods: Participants of the study included 20 overweight men aging 20 - 25 with a body mass index of $25-30$. Having been selected randomly, they were equally divided into control and experimental groups. Three times of blood samplings were done: before the start of training, after the first session of training (acute), and 48 hours after the end of the 8 week training (chronic). Combined endurance and strength trainings were carried out 3 days/week for 8 weeks, as training in the first session started at $50 \%$ of intensity and it was progressively increased by $5 \%$ each week.

Results: There were increases in brain-derived neurotrophic factor levels in the training group after both acute and chronic exercise training. However, the concentrations of brain-derived neurotrophic factor levels and anthropometric characteristics of the subjects were not correlated.

Conclusions: It seems that brain-derived neurotrophic factor levels are affected by physical activity. An increase in brain-derived neurotrophic factors may result in metabolic and physiological changes within the body and have a protective role against inactivity-related diseases such as obesity.
\end{abstract}

Keywords: Combined Training, Brain Derived Neurothrophic Factor, Overweight

\section{Background}

The prevalence of obesity and being overweight are of the biggest public health challenges of the present century and bring about clinical and public health burdens worldwide. Thus, it is acknowledged that national programs for the prevention and treatment of overweight, obesity, and its related comorbidities should be prioritized (1). It is also argued that being one of the most important factors affecting energy metabolism, physical activity has an important role in reducing the risk of obesity and controlling body weight by altering the balance between energy intake and energy expenditure $(2,3)$. Regular physical activities are also beneficial to ones health as they prevent various diseases such as cancer, cardiovascular diseases, diabetes, obesity, and cognitive diseases (4-6).

The neurotrophins, the most important trophic factors in the nerve system, consist of nerve growth factor, brainderived neurotrophic factor, neurotrophin-3, and NT-4/5 (7). Brain-derived neurotrophic factor, first discovered and purified in 1982 from the brain of a pig, plays a major role in neurogenesis and neuronal-death protection, and also positively influences brain plasticity by improving learning and memory (8). Its secreted form has the molecular weight of $27.8 \mathrm{kDa}$ and consists of 247 amino acids (9).

Brain-derived neurotrophic factor in the neural system exerts its biological effects via 2 types of receptors including the pan-neurotropin receptor p75 and the tyrosine kinase receptor B receptor (10). Brain-derived neurotrophic factor expression has been reported in various parts of the human brain including the hippocampus, amygdala, cerebral cortex, cerebellum, septum, and the nucleus of the solitary tract. It also is found in other body tissues such as

Copyright (c) 2016, Annals of Military and Health Sciences Research. This is an open-access article distributed under the terms of the Creative Commons 
the heart, muscles, kidney, lungs, and testes (11).

Aside from its effects on synaptic plasticity in the central nerve system (CNS), it involves elements of cellular energy metabolism and in the periphery it takes part in metabolic processes such as enhancing lipid oxidation in the skeletal muscle via activating adenosine monophosphate-activated protein kinase $(12,13)$. Therefore, it plays a critical role in central and peripheral energy metabolism as studies reveal that brain-derived neurotrophic factor levels are lower in patients with neurodegenerative diseases including Alzheimer's disease and major depression. In addition, obese people and patients with type 2 diabetes have lower levels of brain-derived neurotrophic factor $(14,15)$.

Physical activity is one of the important factors affecting energy metabolism, and brain-derived neurotrophic factor seems to be regulated by exercise and physical activity. However, variations in the types, intensity, and duration of physical activity yield in different physiological responses.

Ruh et al., having investigated the effects of obesity and aerobic exercise training on brain-derived neurotrophic factor, reported that at baseline, the obese group had significantly lower brain-derived neurotrophic factor levels than the normal-weight group; nevertheless, after the exercise training, the increase of brain-derived neurotrophic factor in the obese group was significantly higher than in the normal-weight group. They also concluded that obesity reduces the Brain-derived neurotrophic factor level but exercise training increases its levels (16). Nevertheless, Babaei et al., reported that brain-derived neurotrophic factor levels in athletes are lower than physically inactive people (17).

Regular physical activity is beneficial to health and prevents many diseases; however, using aerobic or resistance exercise programs alone is not sufficient to take advantage of physical activity. Thus, American College of Sports Medicine recommends both of these activities. Therefore, this study investigated the acute and chronic effects of combined training on brain-derived neurotrophic factor levels and the association between brain-derived neurotrophic factor levels and anthropometric variables in overweight young men.

\section{Methods}

\subsection{Subjects}

Volunteers' levels of physical activity and disease records were determined through the special questionnaire. A total of 20 qualified volunteers (overweight, aging 20 - 25 years, and with body mass index 25 - 30) were selected and randomly divided into equal $(\mathrm{n}=10)$ control and experimental groups. It is worth mentioning that all stages of the study accorded the Declaration of Helsinki and were approved with the research ethics committee of sport Sciences research institute with the code of IR.SSRI.REC.1395.109.

\subsection{Anthropometric Measurements}

One week before starting the training in the gymnasium, the participants were briefed on the objectives and methodology of the study and were familiarized with the movements and devices. Their weights, heights, body fat percentages, one repetition maximum (1 RM) of various movements, and maximum oxygen consumptions were also recorded. Specifically, the subjects' heights were measured while standing without shoes against the wall. Their weights were also measured using a digital scale while they had minimal clothing. Their body mass indices were also calculated by dividing weight $(\mathrm{kg})$ by square of the height $\left(\mathrm{m}^{2}\right)$. Their skin fold thicknesses were also measured by caliper according to the Jackson and Pollock method at 3 sites (chest, abdominal, and thigh) in the right side of body to estimate body fat percentage. It deserves mentioning that all measurements were done 3 times in each area by 1 person and the average of 3 measurements was considered as the final record. For the sake of reliability and validity, all measurements were done at the same time, preferably morning (after the overnight fasting).

\subsection{Training Protocol}

This study involved a combined training program (strength and endurance) 3 times a week for 8 weeks. The participants performed 10 minutes of warm up exercises including jogging and stretching movements, and ran for 20 minutes by $50 \%$ to $85 \%$ of maximum heart rate (training started with $50 \%$ of intensity and it was increased by $5 \%$ each week) at the beginning of each session. Then, they performed strength training for large muscles of the upper and lower body, which included lat pull-down, bench press, leg press, barbell shoulder, and knee flexion with $50 \%$ to $80 \%$ of one repetition maximum (training started with $50 \%$ of intensity and it was increased by $5 \%$ each week). They performed 3 sets with 10 repetitions (with 1minute rest interval between sets and 2-minutes rest interval between exercises). Training sessions ended with a 10minute cooling-down though walking slowly (18).

\subsection{Blood Sampling}

In the current study, blood samplings were done at 3 different times including pre-training, after the acute training, and 48 hours after the end of the training period 
(chronic). Blood samples were taken from the antecubital veins of the overnight fasting subjects by a laboratory expert after their sitting on a chair for 15 minutes. The blood samples were then kept at room temperature for 1 hour to be clotted. Then, they were centrifuged and serums were kept at $-80^{\circ} \mathrm{C}$ until final measurements. Brain-derived neurotrophic factor concentrations were measured through the commercially available ELISA kit specific for human samples based on the instruction of manufacturer (EASTBIOPHARM, Hangzhou Co. Ltd, China).

\subsection{Statistical Analysis}

The data were analyzed through the Statistical Package for Social Sciences (SPSS version 16) and the level of significance was determined at $\mathrm{P} \leq 0.05$. As the results of the Kolmogorov-Smirnov tests proved normality of the data, ANOVA $2 \times 3$ with repeated measures and the Bonferroni post hoc test were run for analyzing the data. Independent t-test was also used for testing the differences between the 2 groups in different measurement times. Pearson correlation tests also probbed the relationship between Brain-derived neurotrophic factor and anthropometric variables.

\section{Results}

Table 1 presents means and standard deviations of physical and anthropometric characteristics (including age, height, weight, body mass index, and body fat percentage) of 2 groups.

Table 1. Means and Standard Deviations of Physical and Anthropometric Characteristics of the Subjects $(\mathrm{N}=10)^{\mathrm{a}}$

\begin{tabular}{|c|c|c|c|}
\hline \multirow[t]{2}{*}{ Variable } & \multicolumn{3}{|c|}{ Group } \\
\hline & Measuring & Control & Experimental \\
\hline Age, $y$ & Pre-training & $22.3 \pm 1.5$ & $22.7 \pm 1.5$ \\
\hline Height, $\mathrm{cm}$ & Pre-training & $178.8 \pm 3.1$ & $180 \pm 4$ \\
\hline \multirow{2}{*}{ Weight, kg } & Pre-training & $86.6 \pm 2.6$ & $87.3 \pm 3.9$ \\
\hline & $\begin{array}{c}\text { Post-training } \\
\text { (chronic) }\end{array}$ & $86.7 \pm 2.3$ & $86.4 \pm 3.4$ \\
\hline \multirow{2}{*}{ BMI, $\mathrm{kg} / \mathrm{m}^{2}$} & Pre-training & $27.1 \pm 0.8$ & $26.9 \pm 0.7$ \\
\hline & $\begin{array}{c}\text { Post-training } \\
\text { (chronic) }\end{array}$ & $27.1 \pm 0.9$ & $26.9 \pm 0.6$ \\
\hline \multirow{2}{*}{ Body fat, \% } & Pre-training & $22.8 \pm 1.6$ & $22.6 \pm 1.3$ \\
\hline & $\begin{array}{l}\text { Post-training } \\
\text { (chronic) }\end{array}$ & $22.7 \pm 1.5$ & $21.5 \pm 1.5$ \\
\hline
\end{tabular}

Abbreviation: BMI, Body Mass Index.

${ }^{\mathrm{a}}$ Values are expressed as mean $\pm \mathrm{SD}$.
Differences in anthropometric characteristics of the subjects at different times of measurement, i.e., before and after the training period, were probed through paired ttests, which indicated that only the fat percent in the experimental group was reduced significantly and there were no significant differences in other characteristics of the participants.

The average food intakes of the subjects were recorded 3 days before the blood samplings and analyzed with food analyzer software (NUTRITION 4) (Table 2). It should be mentioned that a copy of the subjects' food intake forms completed in pre training were given to them before the final blood sampling so as to allow their following the forms.

Significant differences were found between brainderived neurotrophic factor levels at different times of measurements $(F=10.68, \operatorname{sig}=0.001)$. There was also a significant interaction between groups and times of measurements $(F=14.53$, sig $=0.001)$.

Table 2. Means and Standard Deviations of Food Intake Analysis $(\mathrm{N}=10)^{\mathrm{a}}$

\begin{tabular}{l|c|c|c}
\hline \multirow{2}{*}{ Variable } & \multicolumn{3}{|c}{ Group } \\
\cline { 2 - 4 } & Stage & Control & Experimental \\
\hline \multirow{2}{*}{ Energy, kca } & Primary & $3187 \pm 143$ & $3241 \pm 179$ \\
\cline { 2 - 4 } & Secondary & $3169 \pm 252$ & $3272 \pm 204$ \\
\hline \multirow{2}{*}{ Carbohydrate, gr } & Primary & $451.1 \pm 58$ & $460 \pm 21.7$ \\
\cline { 2 - 4 } & Secondary & $442.8 \pm 37$ & $455 \pm 33.1$ \\
\hline \multirow{2}{*}{ Protein, gr } & Primary & $129 \pm 48$ & $131.7 \pm 29$ \\
\cline { 2 - 4 } & Secondary & $132 \pm 39$ & $139.8 \pm 36$ \\
\hline \multirow{2}{*}{ Fat, gr } & Primary & $121.1 \pm 19$ & $127.3 \pm 48$ \\
\cline { 2 - 4 } & Secondary & $129.7 \pm 31$ & $120.3 \pm 37$ \\
\hline
\end{tabular}

${ }^{\mathrm{a}}$ Values are expressed as mean $\pm \mathrm{SD}$.

Within group changes for each of the groups were tested through separate ANOVA repeated measures. The control group had no significant difference between the variances of brain-derived neurotrophic factor levels at different times of measurements. However, the brain-derived neurotrophic factor levels of the experimental group were significantly different at different measurement times. The differences were further probed running Bonferroni post hoc test the results of which revealed a significant increase in Brain-derived neurotrophic factor levels after the acute and chronic training, as compared to the pretraining. $(\mathrm{P} \leq 0.05)$ (Figure 1 ).

The differences between the Brain-derived neurotrophic factor levels of the experimental and control groups at different times of measurements were tested through the independent t-test (Table 3). The results 


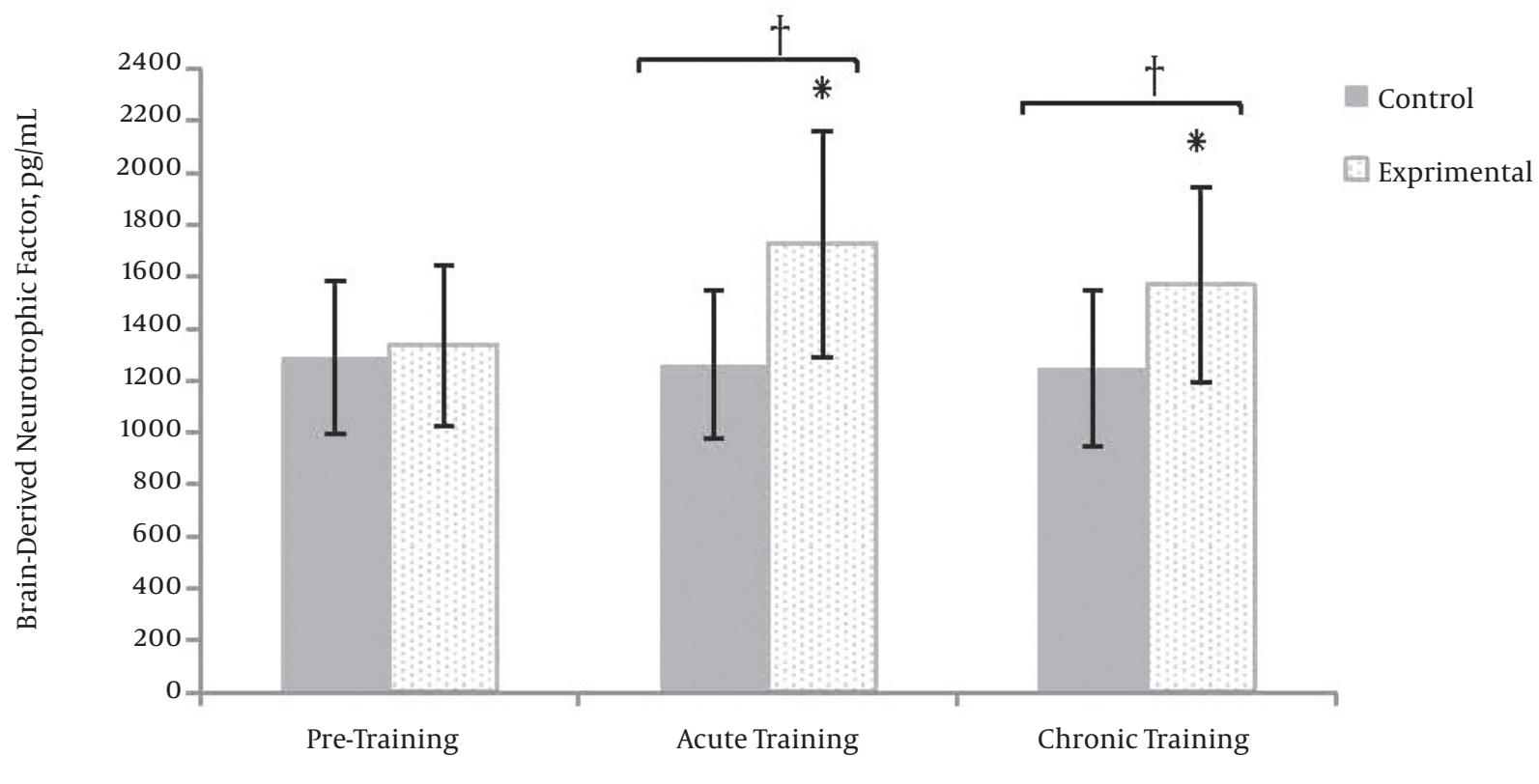

Figure 1. Brain-Derived Neurotrophic Factor Concentrations at Different Times Between the Two Groups

indicated a lack of differences in the concentrations of Brain-derived neurotrophic factor levels of the 2 groups at the pre-training; however, they had different levels of brain-derived neurotrophic factor levels after the acute and chronic training as the experimental group had the higher concentration of brain-derived neurotrophic factor than the control group $(\mathrm{P} \leq 0.05)$.

At the end of training protocol, the relationship between brain-derived neurotrophic factor and anthropometric characteristics including body mass index and fat percentage of the subjects were assessed using Pearson's correlation tests, the results of which revealed no significant correlation between the anthropometric variables and brain-derived neurotrophic factor levels (Figure 2).

\section{Discussion}

This study was carried out to investigate both the acute and chronic effects of combined exercise training on brainderived neurotrophic factor levels and the relationship between brain-derived neurotrophic factor levels and anthropometric variables in overweight young men. Our findings indicated that the acute and chronic combined exercise training (strength and endurance) significantly increased brain-derived neurotrophic factor levels. However, the concentrations of brain-derived neurotrophic factor levels were not related to anthropometric variables including the body fat percentage and body mass index.
Variations in the levels of brain-derived neurotrophic factor due to the various types of physical activities have been investigated and contradictory results have been reported. Some studies reported increased brain-derived neurotropin levels as a result of exercise, which is in line with the findings of this study (19-22). Brain-derived neurotrophic factor can cross the blood-brain barrier in both directions, i.e. from the brain to the periphery and from the periphery to the brain. Furthermore, brain-derived neurotrophic factor levels in the brain is associated with the serum concentration. Thus the serum level of brainderived neurotrophic factors may reflect its brain level (17). During physical activity and resting condition, the brain contributes to approximately $70 \%-80 \%$ of the circulating brain-derived neurotrophic factor levels, and thus acts as its main source. However, other peripheral sources such as platelets, vascular endothelial cells, and skeletal muscles also contribute to the brain-derived neurotrophic factor levels (23).

The increase in the brain-derived neurotrophic factor levels after the acute physical activity can be due to its enhanced release from different tissues including the active muscles and brain. Also, the increase in its levels after the chronic training may be caused by the increase in gene expression and activating the transcription pathways. Therefore, increase in the Brain-derived neurotrophic factor levels, aside from its roles in neurogenesis and brain health, plays a critical role in central and periph- 
Table 3. The Comparison Between the Brain-Derived Neurotrophic Factor of the experimental and Control Groups at Different Times of Measurements

\begin{tabular}{|c|c|c|c|c|c|}
\hline \multirow[t]{2}{*}{ Measurement } & \multicolumn{2}{|c|}{ Leven tests } & \multirow[t]{2}{*}{$\mathbf{t}$} & \multirow[t]{2}{*}{ df } & \multirow[t]{2}{*}{ sig } \\
\hline & $\mathbf{F}$ & sig & & & \\
\hline Pre-training & 0.108 & 0.747 & 0.33 & 18 & 0.74 \\
\hline After acute training & 1.20 & 0.288 & 2.81 & 18 & $0.01^{\mathrm{a}}$ \\
\hline After chronic training & 1.47 & 0.240 & 2.10 & 18 & $0.04^{\mathrm{a}}$ \\
\hline
\end{tabular}

${ }^{\mathrm{a}}$ Significant difference(P $\left.\leq 0.05\right)$.
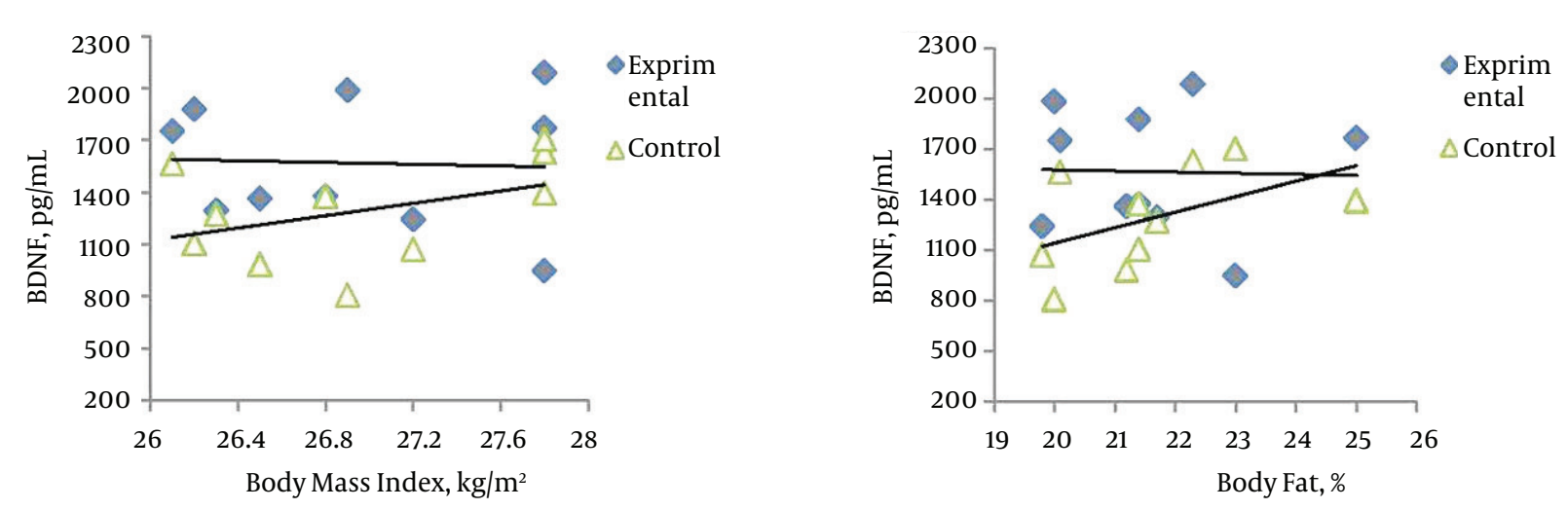

Figure 2. Correlation of Brain-Derived Neurotrophic Factor Levels with Body Mass Index $\left(\mathrm{kg} / \mathrm{m}^{2}\right)$, and Body Fat (\%) for the 2 Groups

eral energy metabolism through hypothalamus pathways.

Inconsistent with our findings, some studies reported a decrease or no changes in the levels of brain-derived neurotrophic factor after physical activity (24-27). This can be due to the effect of type and intensity of exercise, age and physical fitness levels and health status of individuals on the synthesis, as well as release of brain-derived neurotrophic factor into blood stream. In the same vein, Uysal et al. reported that brain-derived neurotrophic factor levels were higher in the voluntary exercise than in the involuntary exercise $(19,22)$. Cho et al. reported that the concentration of brain-derived neurotrophic factor level increased significantly after long term combined exercise in mid-aged women (19). In addition, Schmolesky et al. examined the effects of the intensity and duration of aerobic exercise on brain-derived neurotrophic factor levels and stated that vigorous intensity and long duration exercises caused the greatest elevation in brain-derived neurotrophic factor levels (28).

Also, lack of change in the amount of brain-derived neurotropic factor can also be due to the greater tissue absorption and effective clearance by tissues or transmission into the brain.

Also, some authors reported that reduction in serum brain-derived neurotropic factor reflects a kind of adap- tation to the physical activity, down regulation of brainderived neurotropic factor synthesis, reduction in its releasing mechanism, or more consumption by central nerve system $(17,29)$. In addition, after regular physical activity, plasma volume increases by 10 to $20 \%$, and this may explain the lower levels of brain-derived neurotrophic factor in athletes (17).

Babaei et al. documented that acute aerobic and anaerobic exercises elevated brain-derived neurotrophic factor levels in athlete and sedentary groups as compared to the resting state. Also, the athlete group had lower basal brainderived neurotrophic factor levels than the control group. They suggested that long-term habitual exercise is associated with lower peripheral brain-derived neurotrophic factor levels (17).

As for the relationship between the brain-derived neurotrophic factor levels with anthropometric variables, we found no significant relationships between them. Furthermore, Swift et al., in line with our results, reported that brain-derived neurotrophic factor was not associated with fitness, body composition, and anthropometry in individuals with type 2 diabetes (27). However, Babaei et al. found a positive correlation between brain-derived neurotrophic factor level and body mass index and attributed it to the compensatory metabotropic, rather than a neu- 
rothrophic, role of peripheral brain-derived neurotrophic factor (17).

The increase of brain-derived neurotrophic factor levels following an acute exercise seems to be caused by its elevated release from different tissues into the blood stream on the one hand and its greater tissue absorption on the other hand. In most studies, brain-derived neurotrophic factor levels increased significantly due to acute exercise; however, its concentrations returned to baseline at postexercise, showing a fast disappearance rate of circulating brain-derived neurotrophic factor levels after the cessation of exercise. Similarly, it is reported that brain-derived neurotrophic factor levels decreased below baseline concentration within 3-hours after acute cycling exercise.

\subsection{Conclusion}

Both acute and chronic combined training caused an increase in brain-derived neurotrophic factor levels. Acute exercise may increase the brain-derived neurotrophic factor levels possibly due to the release and secretion of various tissues such as brain or skeletal muscles into blood stream while chronic exercise may enhance its levels by increasing gene expression and activating transcription pathways. Apart from playing important roles in neurogenesis, cognitive functions, and preventing neurodegenerative diseases, increasing levels of brain-derived neurotrophic factors may plays a metabotropic role through the hypothalamic pathway and controls body weight and energy homeostasis in overweight men.

\section{Acknowledgments}

This article has been driven from the first researcher's Ph.D dissertation at Shahid Chamran University of Ahvaz. We hereby acknowledge both the faculties of physical education and sport Science and participants of the study.

\section{Footnote}

Conflict of Interests: None declared.

\section{References}

1. Kelly T, Yang W, Chen CS, Reynolds K, He J. Global burden of obesity in 2005 and projections to 2030. Int J Obes (Lond). 2008;32(9):1431-7. doi: 10.1038/ijo.2008.102. [PubMed: 18607383].

2. Pedersen BK, Febbraio MA. Muscles, exercise and obesity: skeletal muscle as a secretory organ. Nat Rev Endocrinol. 2012;8(8):457-65. doi: 10.1038/nrendo.2012.49. [PubMed: 22473333].

3. Teramoto M, Bungum TJ, Landwer GE, Wagner DR. Association of physical activity to the risk of obesity in adults with physical disabilities. Obes Res Open J. 2015;1(1):16-23. doi:10.17140/oroj-1-104.
4. Aberg MA, Pedersen NL, Toren K, Svartengren M, Backstrand B, Johnsson $\mathrm{T}$, et al. Cardiovascular fitness is associated with cognition in young adulthood. Proc Natl Acad Sci USA. 2009;106(49):20906-11. doi: 10.1073/pnas.0905307106. [PubMed: 19948959].

5. Kruk J. Physical activity in the prevention of the most frequent chronic diseases: an analysis of the recent evidence. Asian Pac J Cancer Prev. 2007;8(3):325-38. [PubMed: 18159963].

6. Blondell SJ, Hammersley-Mather R, Veerman JL. Does physical activity prevent cognitive decline and dementia?: A systematic review and meta-analysis of longitudinal studies. BMC Public Health. 2014;14:510. doi: 10.1186/1471-2458-14-510. [PubMed: 24885250].

7. Lewin GR, Barde YA. Physiology of the neurotrophins. Annu Rev Neurosci. 1996;19:289-317. doi: 10.1146/annurev.ne.19.030196.001445. [PubMed: 8833445].

8. Kuipers SD, Bramham CR. Brain-derived neurotrophic factor mechanisms and function in adult synaptic plasticity: new insights and implications for therapy. Curr Opin Drug Discov Devel. 2006;9(5):580-6. [PubMed: 17002218].

9. Barde YA, Edgar D, Thoenen H. Purification of a new neurotrophic factor from mammalian brain. EMBO J. 1982;1(5):549-53. [PubMed: 7188352].

10. Zoladz JA, Pilc A. The effect of physical activity on the brain derived neurotrophic factor: from animal to human studies. J Physiol Pharmacol. 2010;61(5):533-41. [PubMed: 21081796].

11. Conner JM, Lauterborn JC, Yan Q, Gall CM, Varon S. Distribution of brain-derived neurotrophic factor (BDNF) protein and mRNA in the normal adult rat CNS: evidence for anterograde axonal transport. $J$ Neurosci. 1997;17(7):2295-313. [PubMed: 9065491].

12. Wisse BE, Schwartz MW. The skinny on neurotrophins. Nat Neurosci. 2003;6(7):655-6. doi: 10.1038/nn0703-655. [PubMed: 12830151].

13. Pedersen BK, Pedersen M, Krabbe KS, Bruunsgaard H, Matthews VB, Febbraio MA. Role of exercise-induced brain-derived neurotrophic factor production in the regulation of energy homeostasis in mammals. Exp Physiol. 2009;94(12):1153-60. doi: 10.1113/expphysiol.2009.048561. [PubMed: 19748969].

14. Sakuma K, Yamaguchi A. The recent understanding of the neurotrophin's role in skeletal muscle adaptation. J Biomed Biotechnol. 2011;2011:201696. doi: 10.1155/2011/201696. [PubMed: 21960735].

15. Krabbe KS, Nielsen AR, Krogh-Madsen R, Plomgaard P, Rasmussen P, Erikstrup C, et al. Brain-derived neurotrophic factor (BDNF) and type 2 diabetes. Diabetologia. 2007;50(2):431-8. doi: 10.1007/s00125-0060537-4. [PubMed: 17151862].

16. Roh HT, So WY. The effects of aerobic exercise training on oxidantantioxidant balance, neurotrophic factor levels, and blood-brain barrier function in obese and non-obese men. J Sport Health Sci. 2016:1-7. doi:10.1016/j.jshs.2016.07.006.

17. Babaei P, Damirchi A, Mehdipoor M, Tehrani BS. Long term habitual exercise is associated with lower resting level of serum BDNF. Neurosci Lett. 2014;566:304-8. doi: 10.1016/j.neulet.2014.02.011. [PubMed: 24572590].

18. Atashak $\mathrm{S}$. The effect of the eight-week progressive concurrent training on inflam-matory index of cardiovascular disease predictor, and body composition in sedentary middle-age men [In Persian]. Cardiovasc Nurs J. 2013;2(3):16-25.

19. Cho HC, Kim JK, Lee NJ, Kim SY, Yoon NK. Effects of combined exercise on cardiovascular risk factors and serum BDNF level in mid-aged women. J Exerc Nutrition Biochem. 2014;18(1):61-7. doi: 10.5717/jenb.2014.18.1.61. [PubMed: 25566440].

20. Afzalpour ME, Chadorneshin HT, Foadoddini M, Eivari HA. Comparing interval and continuous exercise training regimens on neurotrophic factors in rat brain. Physiol Behav. 2015;147:78-83. doi: 10.1016/j.physbeh.2015.04.012. [PubMed: 25868740].

21. Bayani H, Fallah Mohammadi Z, Fazelzadeh M. The Acute effect of aerobic and anaerobic exercise on serum levels of BDNF and cortisol in active men [In Persian]. $2014 ; 6$ (11):49-57. 
22. Uysal N, Kiray M, Sisman AR, Camsari UM, Gencoglu C, Baykara B, et al. Effects of voluntary and involuntary exercise on cognitive functions, and VEGF and BDNF levels in adolescent rats. Biotech Histochem. 2015;90(1):55-68. doi: 10.3109/10520295.2014.946968. [PubMed: 25203492].

23. Rasmussen P, Brassard P, Adser H, Pedersen MV, Leick L, Hart E, et al. Evidence for a release of brain-derived neurotrophic factor from the brain during exercise. Exp Physiol. 2009;94(10):1062-9. doi: 10.1113/expphysiol.2009.048512. [PubMed: 19666694].

24. Kim Y. The effect of regular Taekwondo exercise on Brain-derived neurotrophic factor and Stroop test in undergraduate student. $J E X$ erc Nutrition Biochem. 2015;19(2):73-9. doi:10.5717/jenb.2015.15060904. [PubMed: 26244125].

25. Vosadi E, Barzegar H, Borjianfard M. The Effect of endurance training and high-fat diet in brain-derived neurotrophic factor (BDNF) in the male adult rat hippocampus [In Persian]. Arak Univ Med Sci J. 2014;16(10):84-92.
26. Goda A, Ohgi S, Kinpara K, Shigemori K, Fukuda K, Schneider EB. Changes in serum BDNF levels associated with moderate-intensity exercise in healthy young Japanese men. Springerplus. 2013;2:678. doi: 10.1186/2193-1801-2-678. [PubMed: 24386624].

27. Swift DL, Johannsen NM, Myers VH, Earnest CP, Smits JA, Blair SN, et al. The effect of exercise training modality on serum brain derived neurotrophic factor levels in individuals with type 2 diabetes. PLoS One. 2012;7(8):e42785. doi: 10.1371/journal.pone.0042785. [PubMed: 22880108].

28. Schmolesky MT, Webb DL, Hansen RA. The effects of aerobic exercise intensity and duration on levels of brain-derived neurotrophic factor in healthy men. J Sports Sci Med. 2013;12(3):502-11. [PubMed: 24149158].

29. Numakawa T, Kumamaru E, Adachi N, Yagasaki Y, Izumi A, Kunugi H. Glucocorticoid receptor interaction with TrkB promotes BDNFtriggered PLC-gamma signaling for glutamate release via a glutamate transporter. Proc Natl Acad Sci U S A. 2009;106(2):647-52. doi: 10.1073/pnas.0800888106. [PubMed:19126684]. 